\title{
Quaderni
}

QUADERNI Communication, technologies, pouvoir

67| Automne 2008

Jeu vidéo et discours

\section{Jeux des discours normatifs et enjeux de l'analyse}

Avant-propos

Ian Bogost et Olivier Mauco

\section{OpenEdition}

Journals

Édition électronique

URL : http://journals.openedition.org/quaderni/185

DOI : 10.4000/quaderni.185

ISSN : 2105-2956

\section{Éditeur}

Les éditions de la Maison des sciences de l'Homme

\section{Édition imprimée}

Date de publication : 5 octobre 2008

Pagination : 5-10

\section{Référence électronique}

Ian Bogost et Olivier Mauco, « Jeux des discours normatifs et enjeux de l'analyse », Quaderni [En ligne], 67 | Automne 2008, mis en ligne le 05 janvier 2012, consulté le 22 septembre 2020. URL : http:// journals.openedition.org/quaderni/185; DOI : https://doi.org/10.4000/quaderni.185 


\section{$D$ ossier}

avant-propos :

jeux des

discours

normatifs

et enjeux

de l'analyse

Ian

Bogost

Professeur en Digital Media, Georgia Tech

Fondateur du studio Persuasive Game

Olivier

Mauco

A.T.E.R. en Science Politique CRPS, Paris 1
Les jeux vidéo sont des objets polymorphes, entre média audiovisuel, jeu industriel et programme informatique. Les jeux vidéo se distinguent cependant de ces différentes dimensions en mêlant simultanément dans les logiques de l'interaction avec la machine, la posture de spectateur à celle de joueur acteur. Chaque écran où l'interaction est techniquement possible apparait potentiellement comme un espace ludique : télévision, ordinateur, téléphone portable, GPS, etc. Les jeux vidéo sont ainsi des jeux codés informatiquement qui instaurent une relation d'interactivité avec un écran dans un espace de négociation.

Ses origines techniques sont multiples : jeu de bagatelle ${ }^{1}$ et ses versions mécaniques comme le flipper et le patchinko, programme militaire informatique ${ }^{2}$, jeu des Hanafudas, jeu de tir électromagnétique des fêtes foraines. Cette liste non exhaustive des parents du jeu vidéo illustre à la fois le métissage technique et permet de saisir la contemporanéité des discours autour du jeu vidéo. Les influences multiples dans les genres de jeu vidéo ${ }^{3}$ sont en partie des transpositions des représentations socialement construites autour des techniques évoquées précédemment.

La profusion de discours sur les jeux vidéo révèle des importations de conceptions relatives à d'autres domaines d'activité. Ce passage est possible par la conjonction de faits sociaux identifiables et de grilles de lectures soutenues par des experts en concurrence. Exemple archétypale, l'addiction est à la croisée des discours sur le jeu pathologique (jeu d'argent) et des entreprises de définitions de la dépendance à l'Internet, et s'en réfère à l'imaginaire de la toxicomanie. Les 
débats sociaux et politiques autour de la violence, l'addiction, de la liberté économique s'appuient sur les discours accompagnant les médias et jeux antérieurs. Cette vivacité des discours tient à la double dimension ludique et médiatique du jeu vidéo, dans un éternel retour discursif porté par les entrepreneurs de morales, les industriels et les experts, souvent reconvertis et transfuges de domaines de compétence connexes.

\section{Pourtours du jeu vidéo}

Définissons l'objet avant les discours. Marcel Mauss considérait, dans ses réflexions exploratoires, les jeux comme une antichambre des organisations sociales futures: "les jeux sont souvent à l'origine des métiers et de nombreuses activités élevées, rituelles ou naturelles, essayées d'abord dans l'activité de surplus que constituent les jeux. " ${ }^{4}$ Cette fonction sociale du jeu est reprise par les philosophes du jeu : "l'émulation ludique est plus ancienne que la culture même comme impulsion de la vie sociale, et agit comme un ferment sur le développement des formes de la culture archaïque. " ${ }^{5}$ Pour Huizinga, le jeu apparaît comme cet espace clos, coupé de la réalité, où l'activité n'a de sens que dans l'espace du jeu, sans conséquence sur le réel. Cet idéal de liberté dans le jeu, au-delà de tout contrôle autre que conventionnel entre joueurs, tend à négliger les problématiques de production des jeux. L'idéal d'égalité des participants, qui n'est qu'une condition initiale de participation et ne vaut que dans un consentement mutuel des joueurs, ne doit pas faire oublier la structure agonistique, fortement compétitive et hiérarchique du jeu. La liberté réside dans la marge de la règle conventionnellement instituée avec les autres joueurs ${ }^{6}$.
Les vainqueurs forment une oligarchie au sein $\mathrm{du}$ jeu, or en tant que lieu de passage, espace de socialisation, des transferts dans la réalité sont souhaités : le pouvoir politique s'affirme mais avance sous couvert de liberté. Au final, le masque et le vertige permettent un renversement temporaire de l'ordre social dans un processus de régulation et de reproduction de cet ordre. En tant qu'idéal d'expression et de société ${ }^{7}$, le jeu cristallise en son sein les aspirations sociales et politiques. Comme espace utopique, il produit des $«$ discours limites $»^{8}$ et les positionnements des non-joueurs, en tant qu'extérieurs du jeu, produisent en contrepartie des incitations de retour à la normalité.

À la différence des jeux traditionnels produits par le groupe à destination du groupe, en tant que lieu de passage et de socialisation, les jeux vidéo sont un produit industriel créé par un groupe de producteur et à destination d'un public potentiellement mondial (par delà les spécificités des zones économiques de l'industrie : Japon et Asie, États-Unis, Europe).

En tant qu'industrie culturelle ${ }^{9}$, le jeu vidéo fonctionne sur des logiques d'uniformisation des produits (mise en place de licences, déclinaison du même titre sur différentes plates-formes), de massification du public (les chiffes de l'industrie tendant à légitimer la maturité grandissante du public et l'équilibre des genres). En tant qu'industrie du divertissement, les producteurs tentent de produire une expérience unique au joueur, que ce soit de par la nature même d'un jeu vidéo qui offre un domaine des possibles - du jeu dans le jeu -, et les processus de personnification de la mise en scène de soi, notamment avec la création 
d'avatars (des personnages, aux voitures que l'on peut peindre et modifier).

Actuellement, une lutte est en cours en France pour faire entrer le jeu vidéo dans l'une des deux cases mentionnées. En tant qu'industrie culturelle, le jeu vidéo pourra recevoir des aides de l'État (avance sur recette) mais en contrepartie sera réglementé sur le modèle du cinéma ou de l'audiovisuel. En tant qu'industrie du divertissement, le jeu vidéo sera exempt de contrôle mais dépourvu d'aides à la création. Ainsi, les enjeux du jeu vidéo sont multiples : contrôler la production du jeu vidéo, réglementer la distribution, normaliser la pratique afin qu'elle soit acceptée. Ces problématiques s'inscrivent dans différents niveaux : l'économique, le politique et le social.

\section{Résurgences des discours}

L'émergence de nouveaux médias et biens culturels se voit de manière quasi-systématique accompagnée d'un ensemble de discours tendant à qualifier l'importance du phénomène. Du roman pour jeunes filles provoquant l'hystérie ${ }^{10}$ à la bande dessinée illégitime ${ }^{11}$ et subversive ${ }^{12}$, en passant par le cinéma et la télévision, la notion d'effets semble prédominer dans les premières analyses. L'article d'Alexis Blanchet approfondira ce parallélisme des discours de la violence entre ceux accompagnant la naissance du cinéma et ceux du jeu vidéo. Paradoxalement ces entreprises de discréditation des pratiques présentent la particularité de contribuer en même temps à la légitimation de l'importance de l'objet de par la production croissante de critiques, d'un point de vue quantitatif.
Les jeux vidéo, souvent marginalisés et accusés de nombreux maux, n'échappent pas à ce processus. Des associations de défense et de protection de l'enfance ou de victimes de tueries se constituent ou se recyclent en s'emparant du jeu vidéo, dès lors présenté comme responsable de certaines atrocités, à l'image du massacre de Littleton. Les jeux vidéo rendraient violents et seraient la cause d'une addiction forte des plus jeunes joueurs. Cette double accusation renvoie à des thématiques politiques sensibles : la question du monopole de la violence légitime, ici remis en cause par cette pratique illégitime, ou la définition du normal et du pathologique, d'où la tentative de marginalisation des jeux vidéo en érigeant des figures stéréotypées anormales (image du joueur violent ou du joueur drogué). Ces thèses défendues par ces "entrepreneurs de morale ${{ }^{13}}^{13}$ trouvent un certain écho dans les médias généralistes, jusqu'à alerter les pouvoirs publics. L'article d'Olivier Mauco abordera ainsi la question de la construction médiatique des problématiques de la violence et de l'addiction, en cadrant l'analyse autour des contraintes de choix des experts par les journalistes.

La notion même d'addiction relève d'une extériorisation du pathologique. La pratique du jeu vidéo n'est pas en soi addictive, mais les usages excessifs requalifiés comme tels sont avant tout socialement construits et s'inscrivent dans les problématiques de la psychiatrie moderne. L'usage du jeu vidéo est ainsi ambivalent : l'article de Thomas Gaon et Michael Stora nous propose de déconstruire dans un premier temps la notion d'addiction, pour dans un second temps présenter un usage du jeu vidéo comme outil thérapeutique, un moyen de médiation capable 
d'incarner le non-dit. Ce renversement même illustre la dimension polymorphe des jeux et la pluralité des pratiques possibles.

\section{Pour une analyse critique des contenus des jeux vidéo}

La pluralité des discours autour du jeu vidéo souffre d'un certain silence : le contenu même des jeux s'il est incriminé n'est pas pour autant étudié par les différents entrepreneurs. Une analyse approfondie pourrait permettre d'éviter les transpositions des croyances et nuancer les prises de postions. Prenons le cas très particulier de NRA Gun Club, un jeu de tir à la première personne commandé par la National Rifle Association (NRA).

Le jeu contient plus de cent armes à feu, toutes modélisées avec réalisme tant dans l'apparence que dans la mécanique. Les joueurs choisissent parmi une douzaine de défis de tir, de la cible d'intérieur à un champ de ball-trap en extérieur, en passant par un stand de tir de fête foraine. Comme on pouvait s'y attendre, le jeu adopte les conventions du genre jeu de tir à la première personne (FPS). Le joueur est debout derrière l'arme à feu ou vise dans la lunette pour toucher le centre d'une cible.

La plupart des gens et des joueurs, même avides de jeux de FPS, reculeraient brutalement à l'idée d'un jeu autorisé et produit par la NRA. Pour certains, la nature du partenariat est une raison suffisante pour éviter NRA Gun Club. Pour d'autres, la NRA évoque les souvenirs ludiques amers de Deer Hunter et de ses dérivés. Le cynique attentif pourrait noter que l'éditeur du jeu,
Crave Entertainment, a aussi publié The Bible Game, un jeu de plateau biblique pour enfants sur Game Boy et PlayStation 2.

Pourtant, il convient de dépasser les discours critiques des plus sceptiques, les préconceptions, pour s'intéresser au contenu même du jeu, de le juger par rapport à la culture vidéoludique contemporaine. D'une certaine manière, ce jeu illustre la stratégie de plus en plus perfectionnée de l'organisation en termes de communication publique, qui n'en est pas à sa première utilisation d'un jeu vidéo pour promouvoir ses valeurs ${ }^{14}$. Avec NRA Gun Club, l'organisation opère un virage rhétorique important, loin de cette réputation d'association de péquenauds. En fait, au regard du culte des armes à feu dans les jeux vidéo des quinze dernières années, NRA Gun $C l u b$ apparaît comme l'un des jeux sérieux des plus efficaces, tant il coupe court au fantasme et à l'imaginaire des armes à feu, en proposant une simulation de la réalité du tir à armes à feu, allant contre le plaisir même procuré par ces types de jeu.

Le maniement des armes à feu est dans ce jeu un travail ennuyeux, lent, laborieux. La tenue d'une arme à feu réelle n'est pas du tout amusante et, par expérience, est même inquiétante. La réalité de la puissance d'une arme à feu est une sensation accablante et un rappel du sérieux de telles armes. Et pourtant, la représentation d'armes à feu dans la plupart des jeux vidéo est exactement à l'opposé : c'est une célébration, un imaginaire de puissance et d'inconséquence générale (non pas en terme de tir et de meurtre, mais dans le simple fait de manier l'arme). Le jeu en devient très fastidieux. En faisant des armes à feu ennuyeuses, lentes et laborieuse, NRA Gun 
Club pourrait être l'incarnation même du discours tant parodié sur la responsabilité des armes à feu. On pourrait même en tirer que la puissance de la rhétorique $^{15}$ de NRA Gun club est sublimée par l'opposition qu'elle marque avec celle des jeux de tirs traditionnels.

Bien sûr, NRA Gun Club ne dit rien du support fervent de l'organisation de chasse, ni de sa défense souvent absurde du deuxième amendement. Si vraiment des médias violents influencent le comportement du joueur, la NRA et Crave Entertainment peuvent se targuer que NRA Gun Club est un jeu non violent tant il opère en contrepoint avec l'imaginaire des jeux de tirs commerciaux. Le seul plaisir se trouve en grande partie dans la maîtrise des mécanismes. Quand le pouvoir destructif de l'arme produit l'excitation, c'est une excitation particulière dans un cérémoniel contextualisé, empli d'une inquiétude d'autant plus accentuée par la rareté des tirs. Et peut-être est-ce exactement le type d'imaginaire sur les armes à feu dont nous avons vraiment besoin.

Au travers de cet exemple il apparaît nécessaire d'entreprendre une analyse critique du contenu. Il ne s'agit en rien de légitimer les jeux vidéo, de les consacrer par des études analytiques, mais au contraire d'ouvrir les perspectives, de combattre les représentations ordinaires. Sébastien Genvo propose ainsi l'instauration d'une analyse critique du jeu vidéo, la constitution d'une réelle science du jeu, au même titre que les analyses littéraires, cinématographiques et pour d'autres arts. L'émergence d'une telle critique pourrait offrir un espace d'existence pour les créations marginales, les œuvres d'auteurs ne pouvant rencontrer de succès économique, au même titre que le cinéma indépendant ou la littérature sortie des logiques marketing de best-sellers. L'analyse de contenu permet ainsi de mieux saisir les logiques inhérentes à ces jeux, de marquer les différences avec les médias traditionnels. Ainsi Delphine, Grellier dans son approche anthropologique de la mort dans les jeux vidéo, actualise la question de la gestion de la finitude, notamment au travers des différentes stratégies des concepteurs du jeu. La mort est un élément du jeu, et si elle s'en réfère à un imaginaire socialement partagé, elle occupe une place très particulière dans les jeux vidéo.

Enfin l'analyse de contenu des jeux, la connaissance des pratiques, des logiques permettrait aux pouvoirs publics de saisir la pluralité des enjeux. Par delà les discours sur la violence et l'addiction, de véritables défis économiques se jouent actuellement. Ainsi l'enjeu politique de réglementation des jeux vidéo prend une tournure d'autant plus décisive, notamment avec le cas des mondes virtuels, comme les jeux de rôle massivement multi-joueurs (MMORPG) et les mondes synthétiques tels Second Life. L'avenir de ces espaces est potentiellement menacé. La recherche d'Edward Castronova, Travis Ross, Phoebe Elephant et Robert Cornell pose la question de l'opportunité d'une réglementation excessive des mondes virtuels ludiques par les pouvoirs publics. L'ouverture de l'espace ludique, l'effacement progressif de la membrane protectrice apparaît symptomatique de cette érosion permanente du jeu dans les sociétés contemporaines. Ainsi les enjeux économiques de l'interopérabilité, à savoir la possibilité de naviguer entre ces espaces sans coût de transfert, sans perte de ses investissements personnels, pose, d'une part, la question du contrôle centralisé par l'État fédéral américain, 
et d'autre part, l'opportunité économique d'une telle reconfiguration. L'article de Viktor MayerSchönberger entend ainsi explorer ces notions au travers du moment constitutionnel, ce tournant fondamental qui se négocie pour ces mondes numériques.
$\mathrm{N} \cdot \mathrm{O} \cdot \mathrm{T} \cdot \mathrm{E} \cdot \mathrm{S}$

1. Stephen L Kent, The Ultimate history of videogames, 2001, Three River Press.

2. Stephen Kline, Nick Dyer-Witheford, Greig de Peuter, Digital Play, The Interaction of Technology, Culture, and Marketing, 2003, McGill-Queen's University Press.

3. Par exemple la thèse de l'omniprésence de la «masculinité militarisée» défendue par Stephen Kline.

4. Marcel Mauss, Manuel d'ethnographie, 1926, p. 80.

5. Johan Huizinga, Homo Ludens, 1951, TEL Gallimard, p. 280.

6. Colas Duflo, Jouer et philosopher, 1997, PUF, p. 80.

7. Johan Huizinga, op. cit, , 1951, p. 28.

8. Ibid., p. 20.

9. Theodor W. Adorno, Max Horkheimer, La Dialectique de la raison, TEL Gallimard, 1983.

10. Lise Queffelec, «Le lecteur du roman comme lectrice : stratégies romanesques et stratégies critiques sous la Monarchie de Juillet », in Romantisme, 1986, Volume 16, Numéro 53.

11. Luc Boltanski, «La constitution du champ de la bande dessinée », in Actes de la Recherche en Sciences Sociales, 1975, Numéro 1, p. 42.

12. Éric Maigret, «La reconnaissance en demi-teinte de la bande dessinée », in Réseaux, 1994, Numéro 67, p. 116.

13. Howard Becker, Outsiders, 1985, Metaillé, p. 146.

14. NRA Varmint Hunter, Interactive Sports Entertainment \& Marketing, 2004.

15. Voir au sujet de la notion de rhétorique dans les jeux vidéo les recherches de Ian Bogost, notamment Unit Operations, MIT Press, 2006 et Persuasive Games, MIT Press, 2008. 